\title{
Volume-preserving mean curvature flow of rotationally symmetric surfaces
}

\author{
Maria Athanassenas
}

\begin{abstract}
A rotationally symmetric $n$-dimensional surface in $\mathbb{R}^{n+1}$, of enclosed volume $V$ and with boundary in two parallel planes, is evolving under volume-preserving mean curvature flow. For large volume $V$, we obtain gradient and curvature estimates, leading to long-time existence of the flow, and convergence to a constant mean curvature surface.
\end{abstract}

Mathematics Subject Classification (1991). 35K22, 58G11, 53C21.

Keywords. Geometric evolution equations, motion by mean curvature under a volume constraint.

\section{Introduction}

Consider $n$-dimensional hypersurfaces $M_{t}$, defined by a one parameter family of smooth immersions $x_{t}: M^{n} \rightarrow \mathbb{R}^{n+1}$, with $M_{t}=x_{t}\left(M^{n}\right)$. The hypersurfaces $M_{t}$ are said to move by mean curvature, if $x_{t}=x(\cdot, t)$ satisfies

$$
\frac{d}{d t} x(p, t)=-H(p, t) \nu(p, t), \quad p \in M^{n}, t>0 .
$$

By $\nu(p, t)$ we denote a choice of unit normal of $M_{t}$ at $x(p, t)$, and by $H(p, t)$ the mean curvature with respect to this normal. The surface area $\left|M_{t}\right|$ of the hypersurfaces is known to decrease under the flow. So the evolution can be used for obtaining minimal surfaces in the limit, if it converges.

Here we are interested in the evolution of compact hypersurfaces $M_{t}$ enclosing a prescribed volume $V$. In particular, we consider the evolution equation

$$
\frac{d}{d t} x(p, t)=-(H(p, t)-h(t)) \nu(p, t), \quad p \in M^{n}, t>0 .
$$

where $h(t)$ is the average of the mean curvature

$$
h(t)=\frac{\int_{M} H d g_{t}}{\int_{M} d g_{t}},
$$


and $g_{t}$ the metric on $M_{t}$. As initial surface we choose a compact $n$-dimensional hypersurface $M_{0}$, with boundary $\partial M_{0} \neq \emptyset$. We assume $M_{0}$ to be smoothly embedded in the domain

$$
G=\left\{x \in \mathbb{R}^{n+1}: 0<x_{n+1}<d\right\}, \quad d>0,
$$

and $\partial M_{0} \subset \partial G$. The vector $\nu(p, t)$ is the outer unit normal.

The surface area $\left|M_{t}\right|$ is again decreasing under the flow defined by (2) and in addition the enclosed volume is constant (see Section 1). In this case the hypersurfaces can be expected to converge to a surface of constant mean curvature which solves the isoperimetric problem.

The motion of surfaces by their mean curvature (1) was first studied by Brakke [5], using methods of geometric measure theory. Huisken [22] proves that compact, convex initial surfaces without boundary converge asymptotically to round spheres. Gage and Hamilton [17], Grayson [19] study the problem for curves in the plane. In the noncompact case it is shown by Ecker and Huisken in [13] that entire graphs over $\mathbb{R}^{n}$ of linear growth 'flatten out'.

The interesting question of the formation of singularities in the nonconvex case is considered by Huisken [24], Grayson [20], Dziuk and Kawohl [9], and more recently by Altschuler, Angenent and Giga [1], Ecker [12].

For the volume-preserving flow (2), Huisken [23] proves long-time existence if the initial hypersurface $M_{0}$ is compact, without boundary and uniformly convex; eventually the $M_{t}$ 's converge to a round sphere enclosing the same volume as $M_{0}$.

The uniform convexity is crucial for the proof; using a maximum principle for parabolic systems developed by Hamilton in ([21], Theorem 9.1), Huisken shows that uniform convexity is preserved for $t>0$.

The major difficulty in the volume-preserving evolution (2), and its difference to the mean curvature flow (1), is how to control $h$, which introduces a global character to the problem. Parabolic maximum principles, an important tool in the investigation of evolution equations (see [10]), either fail or become more subtle.

In this paper, except for the volume constraint, we have a free boundary. A convexity assumption is not natural. We replace this by assuming the initial surface to be rotationally symmetric, contained in the region $G$ between two parallel hyperplanes and for a start the surfaces $M_{t}$ to intersect $\partial G$ orthogonally at the boundary. The motivation is the fact that in solving the isoperimetric problem using methods of the calculus of variations, the minimizers prove to be surfaces of revolution intersecting the obstacle at a right angle [2], [3].

Mean curvature flow (without a volume constraint) for complete rotationally symmetric surfaces has been studied by Simon [27]. He gives gradient and height estimates and discusses pinch off behaviour. His approach is especially interesting for us. Dziuk and Kawohl [9], Grayson [20] and Altschuler, Angenent and Giga [1] consider the question of developing singularities.

The main theorem we prove is 
Theorem. Assume $V, d \in \mathbf{R}$ to be given and $M_{0} \subset G$ to be a smooth, rotationally symmetric, initial hypersurface which intersects $\partial G$ orthogonally at the boundary and encloses the volume $V$. Then the flow defined by (2) will exist for all times $t>0$ and will converge to the cylinder $C \subset G$ of volume $V$ under the assumption

$$
\left|M_{0}\right| \leq \frac{V}{d}
$$

The paper is organised as follows:

In Section 1 we give some definitions and preliminaries. For 'large' volume (see Lemma 1) we prove in Section 2 that the surfaces do not pinch off. Also in this case $h$ is shown to be bounded. Gradient and curvature estimates (Section 4 and 5) lead to long-time existence and convergence to a constant curvature surface (Section 6).

Rotationally symmetric surfaces of constant mean curvature in $\mathbb{R}^{3}$ are known as the Delaunay surfaces [8]; they are plane, the sphere, the cylinder, the catenoid, the unduloid and the nodoid. In [2] the author proves that among them only the sphere (hemisphere) or the cylinder can be stable in $G$, depending on the volume. The present condition on the volume (see Lemma 1) excludes the existence of unduloids in $G$. The flow can only converge to a cylinder, which affirms the result in $[2]$.

The methods we use here are those introduced by Huisken [22] for the mean curvature flow, and also used for instance in [10], [13], [14], [23].

Mean curvature flow, but not the volume-preserving problem, is also investigated from different points of view. Evans and Spruck [15], [16], Chen, Giga and Goto [6], [7] work with hypersurfaces which are defined as level sets of viscosity solutions of a nonlinear partial differential equation on some domain in $\mathbb{R}^{n+1}$. Regularity results are given in [15], [16], [18], [25], [26].

The author would like to thank Klaus Ecker for his interest and support, and the Department of Mathematics at the University of Melbourne and the Centre for Mathematics and its Applications at the Australian University for their hospitality.

\section{Notations and preliminaries}

Let $G=\left\{x \in \mathbb{R}^{n+1}: 0<x_{n+1}<d\right\}$, for given $d>0$. We denote by $\Pi_{i}, i=1,2$, the two parallel hyperplanes bounding the domain $G$.

The $n$-dimensional manifold $M^{n}$ is assumed to be smoothly embedded in $\mathbb{R}^{n+1}$, compact, with boundary $\partial M$. The one-parameter family of surfaces obtained by the flow is defined by means of the position vector

$$
x: M^{n} \times\left[0, t_{1}\right) \rightarrow \mathbb{R}^{n+1},
$$

where $x$ satisfies the evolution equation (2) above. 
By $M_{t}$ we denote the image $M_{t}=x_{t}\left(M^{n}\right)$ and $M_{0}$ will be a given initial surface. In addition we assume

(i) The hypersurface $M_{0}$ is rotationally symmetric about an axis which intersects $\Pi_{i}$ orthogonally.

We also use the parametrization

$$
\rho_{S}:[0, d] \rightarrow \mathbb{R}
$$

for the generating curve of a surface $S$ of revolution. Actually, the flow preserves rotational symmetry (see Fact 1 below).

(ii) The boundary $x_{t}(\partial M)=\partial M_{t}$ is contained in $\partial G=\cup_{i=1,2} \Pi_{i}$.

(iii) $M_{t}$ intersects $\partial G$ orthogonally at the free boundary; i.e. $\dot{\rho}(z)=0$, for $z=0$ and $z=d$. (Here $\dot{\rho}=\frac{d \rho}{d z}$.)

By $g=\left\{g_{i j}\right\}$ and $A=\left\{h_{i j}\right\}$ we denote the metric and the second fundamental form on $M_{t}$. For the mean curvature and the norm of the second fundamental form we have

$$
H=g^{i j} h_{i j}, \quad|A|^{2}=g^{i j} g^{k l} h_{i k} h_{j l} .
$$

Let

$$
\tilde{C}=g^{i j} g^{k l} g^{m n} h_{i k} h_{l m} h_{n j} .
$$

Latin indices are used for $n$ and Greek for $n+1$ dimensions, if not otherwise specified.

\section{Facts:}

1. The flow preserves rotational symmetry. This is clear from the evolution equation, since the mean curvature and the normal are symmetric.

2. The surface area $\left|M_{t}\right|$ is decreasing. To see this we need the evolution equation of the metric

$$
\frac{d}{d t} g_{i j}=2(h-H) h_{i j}
$$

(compare ([23], Proposition 1.1), [22])

Therefore

$$
\frac{d}{d t} \sqrt{\operatorname{det} g_{i j}}=-H(H-h) \sqrt{\operatorname{det} g_{i j}}
$$

and using the mean value property of $h$

$$
\frac{d}{d t}\left|M_{t}\right|=-\int_{M} H(H-h) d g_{t}=-\int_{M}(H-h)^{2} d g_{t} \leq 0 .
$$

3. The enclosed volume $V$ is preserved. Denote by $E_{t} \subset G$ the $(n+1)$-dimensional set, with boundary in $G$ equal to $M_{t}$. Then the evolution (2) can be extended to a vector field on the whole of $E_{t}$ and by the first variation formula and the 
divergence theorem we have

$$
\begin{aligned}
\frac{d}{d t} V_{t} & =\int_{E_{t}} \operatorname{div} \frac{d x}{d t} d \mathcal{H}^{n+1}=\int_{\partial E_{t}} \frac{d x}{d t} \cdot \nu d \sigma \\
& =-\int_{M}(H-h) d g_{t}=0 .
\end{aligned}
$$

By standard parabolic theory the flow exists for some short time $0<t<t_{1}$. We write also $\left[0, T_{\max }\right)$ to indicate the maximal time interval for which the flow exists.

\section{A. Height estimates}

By purely geometric arguments it is possible to show that if the enclosed volume is sufficiently large, the surfaces do not pinch off. The condition on the volume is such that, in $\mathbb{R}^{3}$, there are no parts of unduloids satisfying it and at the same time intersecting the planes perpendicularly.

We will need the following notation: Given an initial surface $M_{0}$, we denote by $C$ the cylinder with same enclosed volume $V$ as $M_{0}$, and height $d$.

Lemma 1. If $\left|M_{0}\right| \leq \frac{V}{d}$, then there exists $c_{0}>0$ such that

$$
\rho_{M_{t}}>c_{0}, \quad \text { for } 0 \leq z \leq d, t \in\left[0, t_{1}\right] .
$$

Proof. We recall that $\left|M_{t}\right| \leq\left|M_{0}\right|$ for all $t>0$. Let us now assume that there is some $t_{1}>0$ such that $M_{t_{1}}$ pinches off. We project $M_{t_{1}}$ onto the plane $\Pi_{1}$, using $\pi: \mathbb{R}^{n+1} \rightarrow \mathbb{R}^{n}$. Then

$$
\left|M_{t_{1}}\right| \geq\left|\pi\left(M_{t_{1}}\right)\right| \text {. }
$$

By the volume constraint any $M_{t}$ has to intersect the cylinder $C$ at least once. Therefore

$$
\left|\pi\left(M_{t_{1}}\right)\right|>|\pi(C)|=\omega_{n} \rho_{C}^{n}=\frac{V}{d}
$$

and we obtain a contradiction to $M_{t_{1}}$ pinching off if only we choose $V$ appropriately.

Remarks. (i) Knowing the absolute minimizers in $\mathbb{R}^{3}$ (compare [2]), we can easily see that for small enclosed volume $V$ there is no initial surface satisfying the condition of the Lemma. If $V \leq \pi\left(\frac{2}{3}\right)^{4} d^{3}$ we denote by $S$ the (in this case absolute) minimizing hemisphere. Then

$$
|S| \leq|M|
$$


for any surface $M$ with the same enclosed volume, but

$$
|S|>\frac{V}{d}(=|\pi(C)|) .
$$

(ii) In $\mathbb{R}^{3}$ there are no unduloids intersecting the planes $\Pi_{i}$ perpendicularly at the free boundary and satisfying the volume condition of Lemma 1. As is well-known [8], the generating curve of the unduloid is obtained by rolling an ellipse along the $z$-axis, and tracing the path of either its foci. Let the ellipse be parametrized by $\gamma:[0,2 \pi) \rightarrow \mathbb{R}^{3}$, and denote by $a, b \in \mathbb{R}, 0<b \leq a$ its axes and by $l(\gamma)$ its length. Then the condition for the unduloid to intersect the planes at right angles is

$$
k \frac{l(\gamma)}{2}=d, \quad k \in \mathbb{N},
$$

$\frac{k}{2}$ the periods, i.e. $2 a \leq d$. Consider the cylinder of enclosed volume $V=4 \pi d^{3}$ (see assumption of Lemma 1, for $n=3$ ). Its radius is given by $\rho=2 d$. Any unduloid of the same enclosed volume would have to intersect this cylinder. This is not possible for $2 a \leq d$. Thus, assuming $V \geq 4 \pi d^{3}$ (actually, we only need $V \geq \pi d^{3}$ ) the flow will never converge to an unduloid in $\mathbb{R}^{3}$.

(iii) Height estimates from above.

Assume there exists an $R$ such that $\rho_{M_{t}} \geq R$ at some given time $t$. We would then have

$$
\left|M_{0}\right| \geq\left|M_{t}\right|>\omega_{n}\left(R-\rho_{C}\right)^{n}
$$

by comparing the surface area of $M_{t}$ to that of the $n$-dimensional annulus of radii $\rho_{C}$ and $R$. Here $\rho_{C}=\left(\frac{V}{\omega_{n} d}\right)^{1 / n}$ denotes the radius of the cylinder $C$ of enclosed volume $V$. Of course $M_{t}$ has to intersect this cylinder.

We deduce from the above that

$$
R \geq\left(\frac{\left|M_{0}\right|}{\omega_{n}}\right)^{1 / n}+\left(\frac{V}{\omega_{n} d}\right)^{1 / n}
$$

would contradicts the fact that the evolution decreases surface area.

\section{B. Estimates on $h$}

Lemma 2. Assume $M \subset G$ to be a smooth, rotationally symmetric hypersurface, intersecting $\Pi_{i}$ orthogonally at the boundary and with radius function $\rho \geq c_{0}>0$. Then the mean value $h$ of the mean curvature satisfies

$$
0 \leq h \leq c_{1}
$$


where $c_{1}$ depends on the dimension and the height estimates.

Proof. $M$ being rotationally symmetric we have for the mean curvature $H=$ $\kappa_{1}+(n-1) \kappa_{2}$, where $\kappa_{1}$ and $\kappa_{2}$ denote the principal curvatures. If we parametrize $M$ by its radius function $\rho \in C^{\infty}\left([0, d],\left[c_{0}, R\right]\right)$, then clearly

$$
H=-\frac{\ddot{\rho}}{\left(1+\dot{\rho}^{2}\right)^{\frac{3}{2}}}+\frac{n-1}{\rho\left(1+\dot{\rho}^{2}\right)^{\frac{1}{2}}}
$$

and

$$
h=\frac{1}{|M|} \int_{M} H d g=\frac{\int_{0}^{d}\left(-\frac{\ddot{\rho}}{\left(1+\dot{\rho}^{2}\right)} \rho^{n-1}+(n-1) \rho^{n-2}\right) d z}{\int_{0}^{d} \rho^{n-1} \sqrt{\left(1+\dot{\rho}^{2}\right)} d z}
$$

For the second term we have

$$
0 \leq \frac{1}{|M|} \int_{M}(n-1) \kappa_{2} d g \leq c\left(n, c_{0}\right)
$$

For the first we remark that

$$
\frac{\ddot{\rho}}{\left(1+\dot{\rho}^{2}\right)}=\frac{d}{d z}(\arctan \dot{\rho})
$$

and therefore

$$
\begin{aligned}
\frac{n \omega_{n}}{|M|} \int_{0}^{d}-\frac{\ddot{\rho}}{\left(1+\dot{\rho}^{2}\right)} \rho^{n-1} d z & =\frac{n \omega_{n}}{|M|} \int_{0}^{d}-\frac{d}{d z}(\arctan \dot{\rho}) \rho^{n-1} d z \\
& =\frac{n(n-1) \omega_{n}}{|M|} \int_{0}^{d}(\arctan \dot{\rho}) \dot{\rho} \rho^{n-2} d z
\end{aligned}
$$

This is positive, as $(\arctan \dot{\rho}) \dot{\rho} \geq 0$, and bounded

$$
\frac{1}{|M|} \int_{M} \kappa_{1} d g \leq \frac{n(n-1) \omega_{n}}{|M|} \frac{\pi}{2} \int_{0}^{d} \sqrt{1+\dot{\rho}^{2}} \rho^{n-2} d z \leq \frac{(n-1)}{|M|} \frac{\pi}{2} \int_{M} \frac{1}{\rho} d g \leq c^{\prime}\left(n, c_{0}\right),
$$

since $|(\arctan \dot{\rho}) \dot{\rho}| \leq \frac{\pi}{2}|\dot{\rho}| \leq \frac{\pi}{2} \sqrt{1+\dot{\rho}^{2}}$. 


\section{Evolution equations}

Notation. Let $\omega=\frac{\dot{x}}{|x|} \in \mathbb{R}^{n+1}, \hat{x}=\left(x_{1}, \ldots, x_{n}, 0\right)$ denote the unit outer normal to the cylinder intersecting $M_{t}$ at the point $x(p, t)$. Set

$$
v=(\omega, \nu)^{-1}
$$

Remark that $v=\sqrt{1+\dot{\rho}^{2}}$, where $\rho$ is the radius of $M_{t}$, i.e. the "height" of the generating curve. We call $u=\langle x, \omega\rangle$ the height function of $M_{t}$.

Lemma 3. We have the evolution equations

$$
\begin{aligned}
\text { (i) } \quad\left(\frac{d}{d t}-\Delta\right) u=h\langle\nu, \omega\rangle-\frac{n-1}{u}, \\
\text { (ii) } \quad\left(\frac{d}{d t}-\Delta\right) v=-|A|^{2} v+\frac{n-1}{u^{2}} v-2 v^{-1}|\nabla v|^{2}, \\
\text { (iii) } \quad\left(\frac{d}{d t}-\Delta\right) H=(H-h)|A|^{2}, \\
\text { (iv) } \quad\left(\frac{d}{d t}-\Delta\right)|A|^{2}=-2|\nabla A|^{2}+2|A|^{4}-2 h \tilde{C},
\end{aligned}
$$

where $\tilde{C}=g^{i j} g^{k l} g^{m n} h_{i k} h_{l m} h_{n j}$.

Proof. (iii) and (iv) are the same as in [23].

(i) We use the notation $D$ for the gradient in $\mathbb{R}^{n+1}$ and denote by $D \omega$ the $(n+1) \times(n+1)$ matrix of first derivatives of $\omega$. We have

$$
(D \omega)_{\alpha \beta}= \begin{cases}\frac{1}{u}\left(\delta_{\alpha \beta}-\omega_{\alpha} \omega_{\beta}\right) & \text { for } 1 \leq \alpha, \beta \leq n \\ 0 & \text { for } \alpha=n+1 \text { or } \beta=n+1 .\end{cases}
$$

We need the identities

$$
\begin{aligned}
& \langle\nu, D \omega \cdot \nu\rangle=\frac{1}{u}\left(\nu_{i} \nu_{i}-\langle\omega, \nu\rangle^{2}\right)=0, \\
& \langle x, D \omega \cdot \nu\rangle=\frac{1}{u}\left(x_{i} \nu_{i}-\langle\omega, x\rangle\langle\omega, \nu\rangle\right)=0, \\
& D u=\omega \quad \text { and } \quad \nabla^{M} u=\omega-\langle\omega, \nu\rangle \nu .
\end{aligned}
$$

Using (2) we have

$$
\frac{d}{d t} u=-(H-h)\langle\omega, \nu\rangle .
$$


From (1), (3) and with a suitable choice of basis $\left\{e_{i}\right\}_{1 \leq i \leq n}$ for $T_{x} M_{t}$ we obtain

and

$$
\begin{aligned}
\operatorname{div}_{M} \omega & =\operatorname{div} \omega-\langle D \omega \cdot \nu, \nu\rangle=\frac{n-1}{u} \\
\operatorname{div}_{M}(\langle D u, \nu\rangle \nu) & =e_{i}\langle D u, \nu\rangle\left\langle, e_{i}\right\rangle+\langle D u, \nu\rangle \operatorname{div}_{M} \nu=\langle\omega, \nu\rangle H ;
\end{aligned}
$$

therefore

$$
\Delta^{M} u=\operatorname{div}_{M} \nabla^{M} u=\operatorname{div}_{M} \omega-\operatorname{div}_{M}(\langle D u, \nu\rangle \nu)=\frac{n-1}{u}-H\langle\omega, \nu\rangle .
$$

(4) and (6) imply

$$
\left(\frac{d}{d t}-\Delta\right) u=h\langle\nu, \omega\rangle-\frac{n-1}{u}
$$

(ii) As in ([22], Lemma 3.3), we obtain $\frac{d}{d t} \nu=\nabla H$. Therefore, and by (1)

$$
\frac{d}{d t} v=-v^{2}\langle\nabla H, \omega\rangle
$$

The following computation is as in ([4], Prop. 2.1) adapted to our case. For computing the Laplacian of $v$ we will work with normal coordinates in a neighbourhood of the point $x(p, t)$. Also, we will need the relations

$$
\begin{gathered}
\nabla_{e_{i}} \nu=h_{i j} g^{j k} e_{k}, \quad \nabla_{e_{i}} e_{j}=-h_{i j} \nu+\left(\nabla_{e_{i}} e_{j}\right)^{\prime \prime}, \\
{\left[\omega, e_{i}\right]=0,}
\end{gathered}
$$

where " denotes the tangential part of a vectorfield and [, ] the Lie bracket. Then, using (8) and (9), we have

$$
\begin{aligned}
\Delta\langle\omega, \nu\rangle & =e_{i} e_{i}\langle\omega, \nu\rangle \\
& =e_{i}\left\langle\nabla_{\omega} e_{i}, \nu\right\rangle+e_{i}\left\langle\omega, g^{j k} h_{i j} e_{k}\right\rangle \\
& =\left\langle\nabla_{e_{i}} \nabla_{\omega} e_{i}, \nu\right\rangle+h_{i j} g^{j k}\left\langle\nabla_{\omega} e_{i}, e_{k}\right\rangle+e_{i}\left(h_{i j} g^{j k}\left\langle\omega, e_{k}\right\rangle\right) .
\end{aligned}
$$

Having normal coordinates at $x, \mathbb{R}^{n+1}$ being flat, and using (8), (9), as well as the Codazzi equations, for exchanging derivatives of the $h_{i j}$, we obtain 


$$
\begin{aligned}
e_{i}\left(h_{i j} g^{j k}\left\langle\omega, e_{k}\right\rangle\right) & =h_{i j} g^{j k}\left(\left\langle\nabla_{\omega} e_{i}, e_{k}\right\rangle+\left\langle\omega, \nabla_{e_{i}} e_{k}\right\rangle\right)+e_{i}\left(h_{i j} g^{j k}\right)\left\langle\omega, e_{k}\right\rangle \\
& \left.=h_{i j}\left\langle\nabla_{\omega} e_{i}, e_{j}\right\rangle-|A|^{2}\langle\omega, \nu\rangle+e_{j}\left(h_{i j}\right)\left\langle\omega, e_{j}\right\rangle\right)
\end{aligned}
$$

and

$$
\begin{aligned}
\left\langle\nabla_{e_{i}} \nabla_{\omega} e_{i}, \nu\right\rangle & =\left\langle\nabla_{\omega} \nabla_{e_{i}} e_{i}, \nu\right\rangle=\left\langle\nabla_{\omega}\left(-h_{i i} \nu+\left(\nabla_{e_{i}} e_{i}\right)^{\prime \prime}\right), \nu\right\rangle \\
& =-\omega\left(h_{i i}\right)=-\omega\left(h_{i j}\right) g^{i j} .
\end{aligned}
$$

We deduce

$$
\begin{aligned}
\Delta\langle\omega, \nu\rangle & =-\omega(H)+2 h_{i j}\left\langle\nabla_{\omega} e_{i}, e_{j}\right\rangle-|A|^{2}\langle\omega, \nu\rangle+\langle\omega, \nabla H\rangle \\
& =-\omega\left(h_{i j}\right) g^{i j}-h_{i j} \omega\left(g^{i j}\right)-|A|^{2}\langle\omega, \nu\rangle+\langle\omega, \nabla H\rangle .
\end{aligned}
$$

Combining this with (7) we obtain (ii).

\section{Gradient estimates}

General assumption. For the remaining of the paper we always assume the enclosed volume $V$ to be so large that the results of Section 2 hold; i.e. the evolving surfaces do not pinch off and $h$ is bounded.

Remark. Since we have an evolution of rotationally symmetric surfaces, which intersect the hyperplanes $\Pi_{i}$ orthogonally, and $v=\sqrt{1+\dot{\rho}^{2}}$ along the generating curve, the following Proposition 4 gives the gradient estimate.

Proposition 4. Under the assumptions of Section 1 and the large volume of Lemma 1, if we assume $v \leq v_{0}$ on the initial surface $M_{0}$, then

$$
\max _{t>0} v \leq c_{2}\left(n, c_{0}, R, v_{0}\right) .
$$

Proof. Given the Neumann boundary conditions at $z=0$ and $z=d$, we can equivalently consider the evolution of periodic surfaces $\tilde{M}_{t}$ defined along the whole $z$-axis. We assume that the product $u^{2} v$ attains a maximum, denoted by $K$, on $\tilde{M}_{t_{1}}$ for $t_{1}>0$. The idea is to prove that if $K$ is large enough then $\left(\frac{d}{d t}-\Delta\right) u^{2} v \leq 0$ at this maximum point. 
In Lemma 3 we obtained the evolution equations

$$
\begin{aligned}
& \left(\frac{d}{d t}-\Delta\right) v=-|A|^{2} v+\frac{n-1}{u^{2}} v-2 v^{-1}|\nabla v|^{2}, \\
& \left(\frac{d}{d t}-\Delta\right) u=h\langle\nu, \omega\rangle-\frac{n-1}{u},
\end{aligned}
$$

and hence

$$
\left(\frac{d}{d t}-\Delta\right) u^{2}=2 u\left(h v^{-1}-\frac{n-1}{u}\right)-2|\nabla u|^{2}
$$

For the following calculation we remark that

$$
-2 \nabla u^{2} \cdot \nabla v=-2 v^{-1} \nabla v \cdot \nabla\left(u^{2} v\right)+2 v^{-1} u^{2}|\nabla v|^{2} .
$$

We have

$$
\begin{aligned}
\left(\frac{d}{d t}-\Delta\right) u^{2} v= & u^{2}\left(\frac{d}{d t}-\Delta\right) v+v\left(\frac{d}{d t}-\Delta\right) u^{2}-2 \nabla u^{2} \cdot \nabla v \\
= & u^{2}\left(-|A|^{2} v+\frac{n-1}{u^{2}} v\right)+v 2 u\left(h v^{-1}-\frac{n-1}{u}\right) \\
& \quad-v^{2}|\nabla u|^{2}-2 v^{-1} \nabla v \cdot \nabla\left(u^{2} v\right) \\
\leq & -(n-1) v+2 h u
\end{aligned}
$$

at a maximum $K$ of the product $u^{2} v$; in particular, $v \geq \frac{K}{R^{2}}$ at this point, since $c_{0} \leq u \leq R$ (Lemma 1). We also have $0 \leq h \leq c_{1}\left(n, c_{0}\right)$ (Lemma 2).

We deduce that $\left(\frac{d}{d t}-\Delta\right) u^{2} v \leq 0$ at the maximum point whenever

$$
K>\frac{2 R^{3} c_{1}\left(n, c_{0}\right)}{n-1}
$$

Therefore

$$
\max _{t>0} u^{2} v \leq \frac{2 R^{3} c_{1}\left(n, c_{0}\right)}{n-1}
$$

and

$$
\max _{t>0} v \leq \max \left(\frac{2 R^{3} c_{1}\left(n, c_{0}\right)}{(n-1) c_{0}^{2}}, v_{0}\right)
$$




\section{Curvature estimates}

In this section we prove that as long as $M_{t}$ doesn't pinch off and its generating curve is a graph over the $x_{n+1}$-axis with bounded gradient, the curvature and all its derivatives remain bounded as well.

Propositon 5. Under the assumptions of Proposition 4, there exist positive constants $c_{3}\left(n, c_{0}, R, v_{0}\right), c_{4}$ and $c_{5}$, such that the curvature of the evolving surfaces $M_{t}$ is bounded

$$
\max _{t>0}|A|^{2} \leq c_{3}\left(n, c_{0}, R, v_{0}\right)\left(\frac{c_{4}}{\sqrt{c_{5}}}+\frac{1}{c_{5} t}\right), \quad \text { for } t \in\left(0, T_{\max }\right]
$$

Remark. Actually $c_{3}\left(n, c_{0}, R, v_{0}\right)$ depends on $c_{2}\left(n, c_{0}, R, v_{0}\right)$ of Proposition 4 , whereas $c_{5}$ is a technical constant, depending on the chosen testfunction in the proof, and $c_{4}$ depends on the dimension, the height estimates and the bounds on $h$ and $v$, as from Lemma 2 and Proposition 4.

Proof. We proceed as in ([14], proof of Theorem 3.1) and calculate the evolution equation of the product $g=|A|^{2} \varphi\left(v^{2}\right)$, where $\varphi(r)=\frac{r}{1-k r}, k>0$, and $v=\langle\nu, \omega\rangle^{-1}$. The only difference being the volume constraint, which affects the evolution equations of $|A|^{2}$ and $v$ by an additional $-2 h \tilde{C}$ and $\frac{n-1}{u^{2}} v$, respectively, (compare Lemma 3), we end up with the inequality

$$
\left(\frac{d}{d t}-\Delta\right) g \leq-2 k g^{2}-\frac{2 k}{\left(1-k v^{2}\right)^{2}}|\nabla v|^{2} g-2 \varphi v^{-3} \nabla \varphi \cdot \nabla g-2 h \tilde{C}\left(v^{2}\right)+\frac{2(n-1)}{u^{2}} v^{2} \varphi^{\prime}
$$

which replaces (20) of [14].

For estimating the seconds-last term in our case, we use Young's inequality, and obtain

$$
\begin{aligned}
-2 h \tilde{C} \varphi\left(v^{2}\right) & \leq 2 h|A|^{3} \varphi\left(v^{2}\right) \\
& \leq \frac{3}{2}|A|^{4} \varphi^{2}\left(v^{2}\right)+\frac{1}{2} h^{4} \varphi^{-2}\left(v^{2}\right) \\
& =\frac{3}{2} g^{2}+\frac{1}{2} h^{4} \varphi^{-2}\left(v^{2}\right) .
\end{aligned}
$$

We now choose $k>\frac{3}{4}$ and deduce

$$
\left(\frac{d}{d t}-\Delta\right) g \leq-c_{5} g^{2}-a \cdot \nabla g+c_{4}\left(n, c_{0}, c_{1}, c_{2}\right),
$$


where $c_{5}>0$ and $c_{1}$ and $c_{2}$ denote the constants in the bounds on $h$ and $v$ in Lemma 2 and Proposition 4.

By Corollary 1.4 in [11], if $g$ satisfies (1), then

$$
g \leq \frac{c_{4}}{\sqrt{c_{5}}}+\frac{1}{c_{5} t}
$$

on $M_{t}, t \in(0, T]$. The result follows then from the definition of $g$ as the product $|A|^{2} \varphi\left(v^{2}\right)$ and the gradient estimates.

For the higher curvature derivatives we have under the assumptions of Proposition 4

Proposition 6. For each $m \geq 1$ there is $C_{m}$ such that

$$
\left|\nabla^{m} A\right|^{2} \leq C_{m}
$$

uniformly on $M_{t}$ for $0 \leq t \leq T_{\max } \leq \infty$.

Proof. Having obtained uniform bounds on $|A|^{2}$ (Proposition 5) and $h$ (Lemma 2) the proof is a repetition of that of Theorem 4.1 in [23].

Thus, we have long-time existence for the flow:

\section{Corollary 7.}

$$
T_{\max }=\infty
$$

\section{Convergence to surfaces of constant mean curvature}

Having long-time existence for the flow it remains to show that it converges to a constant mean curvature surface as $t \rightarrow \infty$.

Proposition 8. The mean curvature $H$ of the evolving surfaces converges to its average

$$
\sup _{M_{t}}|H-h| \rightarrow 0 \quad \text { as } \quad t \rightarrow \infty
$$


Proof. We note that

$$
\frac{d}{d t}\left|M_{t}\right|=\int_{M}(H-h)^{2} d g_{t}
$$

and therefore

$$
\int_{0}^{\infty} \int_{M}(H-h)^{2} d g_{t} d t \leq\left|M_{0}\right|
$$

Given the uniform estimates on all curvature quantities and their derivatives (section 5), we have that

$$
\int_{M}(H-h)^{2} d g_{t} \quad \text { and } \quad \frac{d}{d t} \int_{M}(H-h)^{2} d g_{t}
$$

are uniformly bounded. Thus, we obtain

$$
\int_{M}(H-h)^{2} d g_{t} \rightarrow 0 \quad \text { for } \quad t \rightarrow \infty .
$$

In order to obtain the result we will now use a standard interpolation argument. We first estimate the supremum norm using the Sobolev inequality for $p>n$ with a constant uniform in time; we then use the a-priori estimates we obtained for the curvature and its derivatives and integration by parts to deduce

$$
\begin{aligned}
\sup _{M_{t}}|H-h| & \leq\left(\int_{M}|\nabla(H-h)|^{p} d g_{t}\right)^{1 / p} \\
& \leq c\left\{\left(\int_{M}(H-h)^{2} d g_{t}\right)^{1 / 2}+\left(\int_{\partial M}(H-h)^{2} d \sigma_{t}\right)^{1 / 2}\right\},
\end{aligned}
$$

which proves the result.

Remark. Having long-time existence, the evolution will eventually converge to an extremum of the surface energy under a volume constraint. The above curvature estimates provide us then with a regularity result for such surfaces.

\section{References}

[1] S. Altschuler, S. Angement and Y. Giga, Mean curvature flow through singularities for surfaces of rotation, preprint.

[2] M. Athanassenas, A variational problem for constant mean curvature surfaces with free boundary, J. Reine Angew. Math. 377 (1987), 97-107.

[3] M. Athanassenas, A free boundary problem for capillary surfaces, Manuscripta Math. 76 (1992), 5-19. 
[4] R. Bartnik, Existence of maximal surfaces in asymptotically flat spacetimes, Comm. Math. Phys. 94 (1984), 155-175.

[5] K. A. Brakke, Math. Notes, Princeton University Press, Princeton, N.Y. 1978.

[6] X. Y. Chen, Y. Giga, S. Goto, Uniqueness and existence of viscosity solutions of generalized mean curvature flow equations, J. Diff. Geom. 33 (1991), 749-786.

[7] X. Y. Chen, Y. Giga and S. Goto, Analysis toward snow crystal growth, to appear in Proceeding of functional Analysis and related topics.

[8] Ch. Delaunay, Sur la surface de révolution dont la courbure moyenne est constante, Journ. Math. pures et appliquées 6 (1841), 309-320.

[9] G. Dziuk and B. Kawohl, On rotationally symmetric mean curvature flow, preprint Nr. 108, SFB 256, Bonn.

[10] K. Ecker, Local techniques for mean curvature flow, Proceedings of the Centre for Mathematics and its Applications, Australian National University 26 (1991), 107-119.

[11] K. Ecker, On mean curvature flow of spacelike hypersurfaces in asymptotically flat spacetimes, J. Austral. Math. Soc. (series A) 55 (1993), 41-59.

[12] K. Ecker, An $\epsilon$-regularity theorem for mean curvature flow of surfaces in 3-manifolds, to appear.

[13] K. Ecker, G. Huisken, Mean curvature evolution of entire graphs, Annals of Math. 130 (1989), 453-471.

[14] K. Ecker and G. Huisken, Interior estimates for hypersurfaces moving by mean curvature, Invent. Math. 105 (1991), 547-569.

[15] L. S. Evans and J. Spuck, Motion of level sets by mean curvature I, J. Diff. Geom. 33 (1991), 635-681.

[16] L. S. Evans and J. Spuck, Motion of level sets by mean curvature II, to appear.

[17] M. Gage and R. S. Hamilton, The heat equation shrinking convex plane curves, J. Diff. Geom. 23 (1986), 69-96.

[18] Y. Giga and S. Goto, Geometric evolutions of phase boundaries, preprint.

[19] M. Grayson, The heat equation shrinks embedded plane curves to round points, J. Diff. Geom. 31 (1987), 285-314.

[20] M. Grayson, A short note on the evolution of a surface by its mean curvature, Duke Math. J. 58 (1989), 555-558.

[21] R. S. Hamilton, Three-manifolds with positive Ricci curvature, J. Diff. Geom. 17 (1982), 255-306.

[22] G. Huisken, Flow by mean curvature of convex surfaces into spheres, J. Diff. Geom. 20 (1984), 237-266.

[23] G. Huisken, The volume preserving mean curvature flow, J. Reine Angew. Math. 382 (1987), $35-48$.

[24] G. Huisken, Asymptotic behaviour for singularities of the mean curvature flow, J. Diff. Geom. 31 (1990), 285-299.

[25] T. Ilmanen, Generalized flow of sets by mean curvature on a manifold, preprint.

[26] T. Ilmanen, The level set flow on a manifold, preprint.

[27] M. Simon, Mean curvature flow of rotationally symmetric surfaces, B.Sc. Thesis, Dept. of Math. ANU, Canberra.

Maria Athanassenas

Department of Mathematics

University of Melbourne

Parkville, VIC 3052

Australia

e-mail: athanas@gizmo.maths.monash.edu.au

(Received: September 27, 1995) 